\title{
DISTRIBUCIÓN ESPACIAL DE LA VEGETACIÓN EN LA SERRA DE SANTA POLA
}

\author{
J. A. Marco Molina
}

Esta aproximación al paisaje vegetal actual de la Serra de Santa Pola intenta relacionar las comunidades vegetales con la intensidad y grado de transformación que ha podido experimentar este elemento del entorno. El pastoreo, la agricultura, el asentamiento humano en definitiva, son los motores más directos de cambios paisajísticos que en ocasiones pueden ser irreversibles.

En un esquema hipotético, comparable con los utilizados para otras actividades humanas, la máxima intensidad de la degradación está relacionada con la distancia y necesidades de los núcleos de población. El espacio más cercano a ellos es el primero y el que más acusadamente sufre las consecuencias, disminuyendo tanto el grado como el momento de la alteración con la distancia y la accesibilidad.

En una topografía como la de la sierra, los sectores menos accesibles, con mayores pendientes y de difícil aprovechamiento económico, son las laderas de las gargantas fluviocársticas que conforman la mayoría de los barrancos, y en menor medida sus cauces. Ante este inconveniente, el esquema de degradación encontraría allí los sectores menos proclives a la misma. Es por tanto lógico pensar que de quedar restos de comunidades vegetales próximas a la natural o clímax se hallen allí.

\section{I.- CONDICIONES FÍSICAS DEL ENTORNO}

Tanto el clima como los suelos y la topografía inciden directamente en la composición y articulación del paisaje vegetal. El relieve es contrastado, ya 
que frente a extensas superficies aplanadas y suavemente inclinadas que caracterizan las partes más altas, están los abruptos escarpes que miran hacia el mar, sobre todo en las inmediaciones del Faro, o en algunas paredes de los cauces de los barrancos. De estas forma, predominan las primeras, que son a la vez las más accesibles.

Por otro lado, la composición eminentemente caliza de la sierra favorece la morfología típica de este roquedo en el que aparecen lapiaces, gargantas e incluso dolinas. Frente a la aparición de la caliza directamente o a la presencia de una delgada capa de «suelo», es de resaltar que los taludes asociados a los escarpes son otro tipo de substrato que puede introducir modificaciones en el poblamiento vegetal. En definitiva, son suelos o terrenos básicos con una componente calcárea que es decisiva fitogeográficamente por imponer una selección de tipo edáfico en las especies que los colonicen.

El clima es un factor decisivo en la distribución de la vegetación; el análisis del total anual medio de precipitaciones (Elx, 1955-1983), es de 278,1 mm., con un ritmo estacional en el que los meses estivales se caracterizan por una indigencia pluviométrica muy acusada, acompañada a su vez por las temperaturas más elevadas, con valores de la evapotranspiración potencial superiores a $150 \mathrm{~mm}$. en los meses de julio y agosto. Estas condiciones imponen un período de paro vegetativo determinado por la aridez, hasta el punto de que en función del análisis del balance hídrico, las condiciones climáticas se definen como las propias de un dominio árido, mesotérmico y con un acusado déficit hídrico (EB'3 $\left.\mathrm{db}^{\prime} 4\right)$. Por tanto, la vegetación tiene que adoptar unas medidas encaminadas a salvar la dificultad que el régimen hídrico impone.

Ante todos estos condicionantes físicos, una comunidad vegetal determinada (léase la clímax), sin ninguna otra interferencia (la antrópica) podría mantenerse. Prueba de ello son los restos encontrados, pero la suma a todas las limitaciones del medio de la actuación humana, provoca una crisis que rompe el frágil equilibrio existente, iniciándose la regresión.

\section{II.- LA ACTUACIÓN ANTRÓPICA}

Además de los actuales espacios agrícolas, los indicios más sobresalientes del aprovechamiento del suelo se hallan en la perduración de los aterrazamientos con muretes de piedra que jalonan los recorridos de gran parte de los barrancos y que hoy están abandonados, pero que han contribuido en el mantenimiento de parte del suelo; excepción hecha de aquellos en los que el deterioro ha provocado, en no pocos casos, su ineficacia en este sentido.

La huella del pastoreo, al menos a tenor de ciertas reminiscencias, es más profunda. Para comprender esta cuestión basta referirse al hecho de que en la toponimia urbana hay una calle con el nombre de Ganaderos, o la presencia de rebaños estables actualmente. A ello se une el que se haya comprobado cómo la trashumancia entre sectores del interior y la costa se mantenga en nuestros días. Tal vez sea ésta una actividad que ha afectado más prolongada e in- 
tensamente, ya que atañe a lugares en los que la agricultura no llega, o que ha influido en el amplio desarrollo de un cultivo arbóreo dedicado al forraje como es el algarrobo.

Igual incidencia han debido tener actividades tales como la tala para la combustión o la construcción, la función militar adscrita a la Intendencia de Marina (no cuantificada) que se remonta a mediados del siglo XVIII, y la reciente expansión de los espacios urbanos motivados fundamentalmente por el desarrollo de una función turística.

Actividades de signo contrario a las anteriores son las repoblaciones forestales que si bien fueron reclamadas por algunas mentes preclaras desde muy antiguo, una demanda oficial referida a la Provincia de Alicante se remonta al siglo XIX, momento en el que se dirige un informe a la Reina Isabel II en el que se habla de esta necesidad ${ }^{1}$. Esta denuncia no encuentra pronta contestación, y habrá que esperar, prácticamente, hasta el primer tercio del siglo actual, para que ésta se produzca.

Las consecuencias tanto de unas actuaciones como de las otras tienen un esquema evolutivo que se podría resumir así: el porcentaje de recubrimiento del suelo por la vegetación natural o clímax es, por regla general, mayor que el de las formaciones subseriales fruto de la degradación. Consiguientemente, la tala, aclaramiento o destrucción total de dicha vegetación tiene una consecuencia inmediata, la desprotección del suelo sobre el que ésta se asentaba. El medio físico podía tener antes de la actuación un cierto equilibrio, frágil, pero que con la desaparición de la cubierta vegetal, el agua, e incluso el viento se cebaron en la erosión y ablación del suelo. A su vez, este encadenamiento de hechos implica la casi imposibilidad de recuperación natural o espontánea de la misma, y los restos de ella, junto a las etapas regresivas, se ven acompañadas después por las especies objeto de las repoblaciones, fruto de las cuales se produce el desarrollo extremado del Pinus halepensis, que pasa a dominar el espectro vegetal, ya no sólo como consecuencia de la actuación antrópica, sino también por su mayor frugalidad.

\section{III.- CATEGORÍAS VEGETALES Y SU DISTRIBUCIÓN ESPACIAL}

Como se apuntaba al principio de este análisis, el objetivo principal es la corroboración de una hipótesis de trabajo referida al proceso de degradación sufrido por el manto vegetal de la Serra de Santa Pola. Para ello la metodología empleada se basa esencialmente en la definición y jerarquización de diferentes categorías o agrupaciones y su distribución espacial en el territorio, de forma que la cúspide la ocupe aquella comunidad o elementos que suponen una mayor proximidad a la vegetación teóricamente natural, primitiva o clímax.

1 Archivo de la Excma. Diputación Provincial de Alicante: Boletín Oficial de la Provincia de Alicante, n. ${ }^{\circ} 171,1868$. p. 2. 
La definición de esta premisa de partida, presenta varias dudas, aunque se tratará de solventarlas convenientemente. La categoría vegetal superior a considerar es el bosque esclerófilo mediterráneo, representado en la provincia de Alicante por el grado Quercus ilex Schmit, en el que la encina es el árbol más característico ${ }^{2}$. La existencia de plantas consideradas por A. RIGUAL MAGALLÓN como indicadoras más frecuentes del clímax de este grado ${ }^{3}$, inducen a pensar que son los restos de la comunidad que ocupa la cúspide aludida, y que las especies que reciben esta consideración observadas son la Quercus coccifera, Juniperus oxycedrus, Arbutus unedo, Daphne gnidium, Smilax aspera y Rubia peregrina, de las que la práctica totalidad se localizan en los lugares resguardados y poco accesibles como son las laderas laterales de los barrancos. Dentro de este grado se incluiría también el recuerdo de una encina que los lugareños sitúan allí donde hoy están las excavaciones del Portus Illicitanus, árbol que fue arrancado.

En el escalón inferior de la jerarquía se consideran las especies más abundantes del subgrado Pistacia lentiscus-Quercus ilex ${ }^{4}$, entre ellas se han observado la Pistacia lentiscus, Quercus coccifera, Ceratonia siliqua, Rhamnus lycioides, Pinus halepensis, Olea europea ssp. sylvestris, Chamaerops humilis, Ephedra fragilis y Asparagus albus. Su distribución espacial no es tan estricta a los barrancos y lugares menos accesibles como en la anterior, aunque es en esas localizaciones en las que su representación es más densa y frecuente. No hay que olvidar que este subgrupo es una variante del grado anterior y que se caracteriza «por un clima más térmico, generalmente ausente de heladas $\rangle^{5}$.

Las consideraciones hechas no son más que una aproximación al estado actual de la vegetación, es necesario pues, un análisis más profundo de las comunidades que integran el espectro vegetal del área para determinar así con más precisión el grado y consecuencias de la degradación y transformación sufridas, ya que si hasta el momento se vislumbran componentes de comunidades con notable valor biológico e impacto paisajístico, también existen las que ocupan las partes más bajas de la jerarquía e indican procesos y condiciones a tener en cuenta.

Antes de pasar a ello conviene reflexionar acerca de unas ideas referidas a la vegetación clímax, ya que en los estudios realizados, y en la mayoría de los casos, la comunidad a la que se confiere esta calificación es la asociación Chamaeropideto Rhamnetum, según apreciaciones hechas por RIVAS GODAY y FOLCHI GUILLEN, que al afirmarlo a la escala que lo hacen, obvian variaciones derivadas de la topografía y exposición que influyen de forma decisiva en la instalación de los manchones integrados por la asociación Querceto len-

2 RIGUAL MAGALLÓN, A.: Flora y vegetación de la Provincia de Alicante. Instituto de Estudios Juan Gil Albert, Diputación Provincial de Alicante, 1984. p. 23.

3 Ibídem: Op. cit. pp. 23-24.

$4 \quad$ Ibídem: Op. cit. pp. 24.

5 Ibídem: Op. cit. pp. 24. 


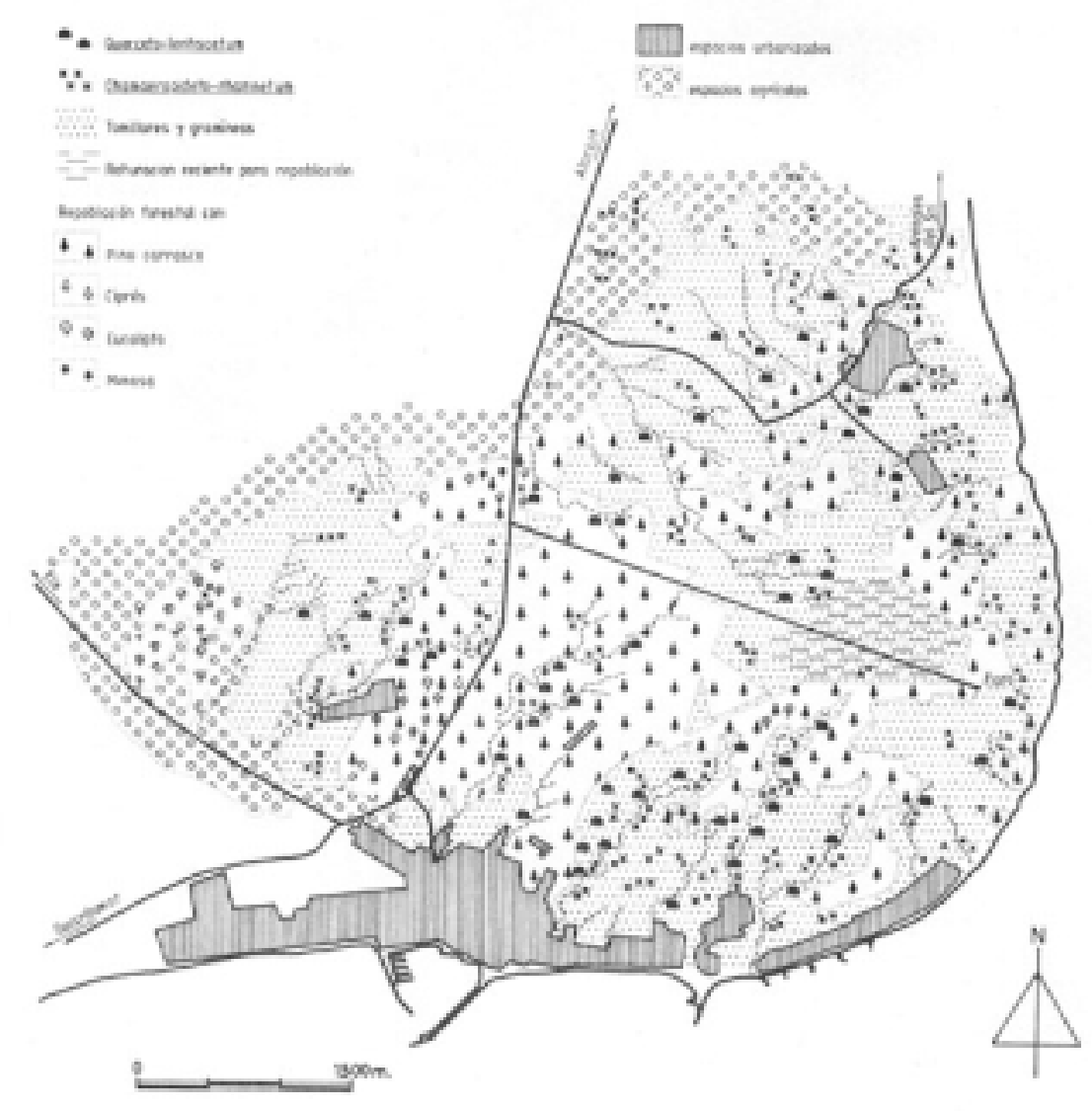

tiscetum, especialmente en los barrancos, que apuntan a una matización de ese planteamiento, ya que en algunos lugares la clímax no sería otra que esta última asociación citada, cuyo dominio se extendería a modo de galerías siguiendo el trazado de los barrancos.

\section{a) El matorral de la asociación Querceto lentiscetum}

El carácter fragmentario y poblamiento discontinuo de este matorral típicamente mediterráneo salpica algunos sectores no sólo de los barrancos, sino también de esas superficies aplanadas que culminan la sierra. De todos modos su distribución es más frecuente en los primeros, deparando un paisaje constituido por manchones más bien reducidos que destacan sobre el substrato abió- 
tico por su intenso color verde, con unos tupidos ramajes, y gran porcentaje de cubrimiento del suelo en el que se asientan, pues su crecimiento es mayor en la horizontal que hacia arriba, sobrepasando en raras ocasiones los dos metros de altura.

Las especies más características son el lentisco y la coscoja, y forman el estrato arbustivo superior al que acompañan frecuentemente el Daphne gnidium y el Brachypodium ramosum, aunque este último no tiene una filiación determinada, y prácticamente es omnipresente en la sierra, pero en esta garriga forma un estrato herbáceo que en ocasiones es muy tupido. Otra especie con cierta relevancia y que viene a complicar la maraña que en ocasiones llega a formar el matorral es una enredadera, la Smilax aspera.

Está claro que su ubicación en los barrancos (quede constancia de un topónimo elocuente: Barranc de la Canyda de la Bellotera ${ }^{6}$ ) demuestra que se aprovechan las condiciones microclimáticas favorables que allí encuentran para su desarrollo, de ahí la escasa representación en puntos con exposición hacia el mediodía o allí donde los sistemas de pendientes no introducen variaciones que amortigüen la notable aridez.

También se ha observado con casi igual localización las especies características de la alianza Oleo Ceratonion tales como el ullastre o acebuche (Olea europea ssp. sylvestris) o el algarrobo (Ceratonia siliqua). El primero es muy escaso y de porte muy reducido, junto a formaciones de Querceto lentiscetum, mientras que el segundo es más abundante, sobre todo cultivado, y algunas veces con un carácter espontáneo. Dentro de esta misma alianza en la que se incluye este matorral hay otra asociación en la que recae de forma más unánime el calificativo de clímax:

\section{b) El Chamaeropideto Rhamnetum}

Este matorral típico de los llanos litorales meridionales de la provincia de Alicante, presenta una diferencia muy notable respecto del anterior: su poblamiento, al menos hoy en día, es muy diseminado, con individuos muy separados entre sí, y con una menor proporción de cobertura del suelo allí donde aparece. Fisionómicamente los integrantes de esta asociación también son muy distintos; los más característicos son el espino negro (Rhamnus lycioides), arbusto de pequeño porte y que confiere al paisaje una tonalidad grisácea, sin resaltar en el terreno; y el palmito (Chamaerops humilis), pequeña palmera de un verde oscuro intenso que lo hace destacar a modo de bolas más o menos esféricas que incluso alcanzan los 3 metros de altura.

A pesar de que también está en los barrancos, en alguno de ellos muy abundantemente (un topónimo significativo es el de Barranc dels Margallons,

6 MARCO MOLINA, J. A.: «La utilitat dels topònims: el cas de la Serra de Santa Pola», en la Rella n. $^{\circ}$ 4, València, Primavera 1985, p. 33. 
en castellano «de los Palmitos») ${ }^{7}$, los restos de esta asociación y su distribución no está tan sujeta a ellos como en el caso anterior, y, aparece también en los interfluvios y en el talud al pie del escarpe de la Serra de Santa Pola orientado hacia el mar.

En las superficies llanas culminantes de la sierra, es frecuente que se interrumpa el paisaje pelado de las mismas por la presencia de algún palmito, y de forma más difícilmente perceptible, por algún espino. Esta ubicación y la separación entre los componentes de la asociación inducen a que ocuparía mayor extensión, pero que se ha visto reducida hasta tales extremos por la actuación secular del hombre. El que esté más extendida que el Querceto lentiscetum tiene su fundamento en el hecho de que se trata de especies más heliófilas y mejor adaptadas a la aridez.

Este matorral, y especialmente el palmito, ha sido objeto del aprovechamiento del hombre, ya sea a través de su eliminación en busca de brotes más tiernos para el ganado, mediante la roturación de espacios agrícolas, o bien para la fabricación de escobas con sus hojas. El resultado ha sido el acantonamiento de la mayor parte de este matorral a los lugares menos accesibles y supervivencia de forma muy fragmentaria en el resto del terreno, siendo frecuente en las lindes de parcelas o en muretes de los aterrazamientos.

En los barrancos, y en contraposición con lo que ocurría con el lentisco y la coscoja, prefiere las laderas bien soleadas y orientadas hacia el S., en las que las sombras del amanecer y del crepúsculo no tienen tanta incidencia. Hay pues en ellos un reparto desigual entre estas vertientes y las orientadas hacia el N. o los fondos de los barrancos. Por otro lado, en casi ninguna ocasión forman parte del subvuelo del pino carrasco, al menos cuando éste tiene un carácter espontáneo, ya que como se verá, la mayoría de estos árboles son producto de replantaciones, y por tanto su aparición allí no tendrá ninguna causalidad fitosociológica.

\section{c) Los tomillares y otras etapas subseriales de la degradación}

En el esquema regresivo se han sucedido formaciones vegetales que tanto desde su categoría biológica, como de su impacto en el entorno han evolucionado negativamente. Si el grado de cobertura del suelo de las mismas se veía reducido notablemente, se llega al punto extremo de máxima desnudez de la superficie terrestre del ámbito analizado (excepción hecha de los peñascos calizos). Hay una clara correlación entre estos poblamientos y el sistema abiótico en el que difícilmente enraízan, ya que los tomillares y las praderas de gramíneas ocupan los suelos más raquíticos.

El tomillar se define como un matorral seco, de ocupación del terreno muy rala, caracterizado por su morfología como una formación a base de plantas camefitas de porte muy reducido, en forma de pequeñas bolas de colo- 
ración grisácea que por coincidir con la del substrato lítico, coadyuva perceptivamente a la identificación de su dominio con un paisaje prácticamente pelado. Aquí se incluyen las especies de la alianza Thymo Siderition O. de Bolos $1957^{8}$, entre las que destacan el tomillo, Anthyllis terniflora y el esparto.

Por su distribución espacial compartida, es necesario describir aquí lo que se ha venido en llamar la pseudoestepa de gramíneas, donde la mayoría de las plantas son anuales o bianuales, acogidas morfológicamente a la definición de plantas terófitas, y componentes de esa masa herbácea que es claro exponente de la degradación y aridez.

En estas formaciones hay además especies como el Brachypodium ramosum o el Sedum sediforme; perfectamente adaptados a los condicionantes xéricos como lo demuestran las hojas suculentas de este último. Una especie de mayor impacto visual dentro de los tomillares y la pseudoestepa es el esparto (Stipa tenecissima), cuyas atochas aparecen salpicando el erial. Esta es otra planta que ha sido aprovechada durante mucho tiempo para la obtención de fibras e hilos (hacer hilito: fer filet, como dicen los santapoleros). Junto a éstas sobresale la bufalaga marina (Thymelaea hirsuta), no sólo por su abundancia, sino también porque se ha observado, que tras el abandono de una parcela, las gramíneas son las primeras que aparecen, y seguidamente lo hace esta especie. Y suele ser en estos casos cuando se ha comprobado la invasión de aquellos lugares por algún pino carrasco.

El ciclo anual de las gramíneas es muy llamativo al romper el casi inmovilismo estacional de las formaciones perennes; así, permanecen bajo la forma de semillas para después germinar en la estación favorable, formando entonces herbazales que incluso llegan a los $50 \mathrm{~cm}$. de altura, con especies como la Avena fatua o la Avena bromoides. Otras especies resultan más llamativas a los sentidos, como las amapolas, las del género Euphorbia, o los gamones (plantas geofitas), además de algunos cardos.

Como es de suponer, ocupan los terrenos culminantes más soleados y donde predomina el caliche o la caliza, ambos escasamente alterados, en los sectores más accesibles y por tanto sujetos a la degradación.

\section{d) Los pinares y otros poblamientos antropogénicos}

Se incluyen bajo este epígrafe las formaciones que tienen su génesis directamente en la actuación antrópica. Hay casos que son muy evidentes como el eucalipto, el ciprés o la mimosa; pero en lo referente al pino carrasco ofrece más dudas. Es sabido que el pino está presente en la vegetación natural del dominio mediterráneo, pero lo que no está tan claro es que pueda representar el clímax o que su extraordinaria difusión sea enteramente natural.

A pesar de estos juicios a priori, el pinar suele contemplarse por parte de algunos grupos como una vegetación natural. Tal vez esa concepción, al me-

8 RIGUAL MAGALLÓN, A.: Op. cit. p. 203. 


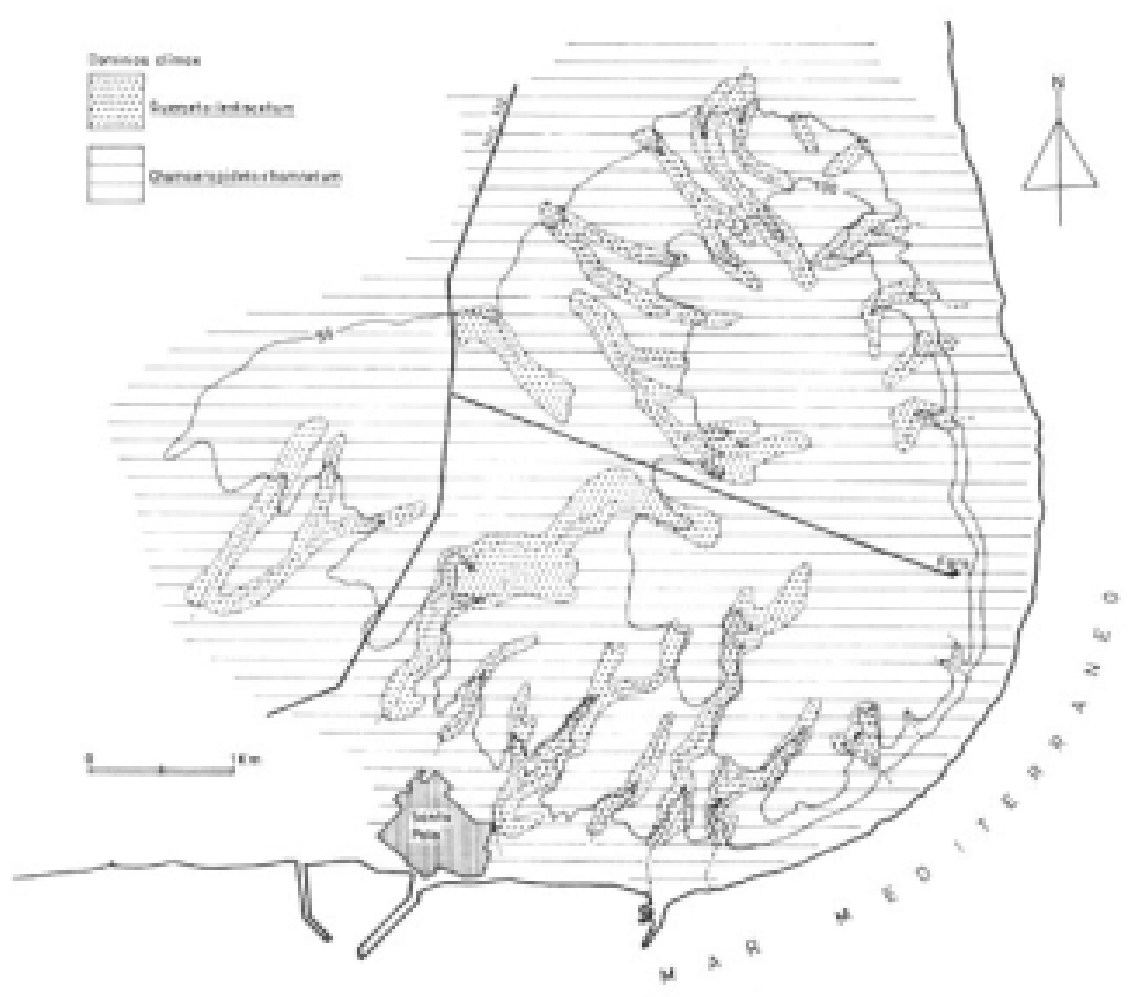

nos en el caso de la Serra de Santa Pola (o ámbitos cercanos como las dunas de Guardamar), se deba a que no han observado el terreno desde el aire, o bien desde el suelo con cierto detenimiento. Efectivamente, la revisión de los fotogramas aéreos denuncian el origen y causa de los pinares en un elevado porcentaje. Se aprecian allí las ocupaciones del terreno en manchas geométricas, con límites bien definidos y rectilíneos tan raros en la naturaleza, e incluso las alineaciones de pinos; se trata pues de una repoblación efectuada en sucesivas etapas y con métodos bien distintos.

A grandes rasgos se diferencia una primera etapa posbélica fruto de la situación coyuntural en la que los mismos habitantes eran los encargados de plantar los pies: a esta etapa corresponden al menos teóricamente los pinos más crecidos y cuya plantación es más irregular. A medida que nos acercamos hasta la actualidad, esta actividad repobladora se ha ido sistematizando hasta el punto de que hoy se utilizan tractores tan potentes que son capaces de arar el caliche, y son plantaciones más regulares y fácilmente diferenciables.

Queda dicho ya que la especie protagonista de esta actuación es el ya citado Pinus helepensis, con un pequeño porcentaje de otras especies menos afor- 
tunadas en su adaptación o menos beneficiosas incluso. De todos modos, a pesar de que en algunos sectores el pino no sobrepasa el porte arbustivo después de muchos años, en otros, prospera adecuadamente, hasta el punto de que también suele aparecer de forma espontánea, aspecto éste que marca una mayor adaptación edafoclimática.

\section{IV.- CONCLUSIONES}

La comprensión y explicación de la distribución espacial de las especies, comunidades y dominios vegetales, no puede, ni debe, disociarse de una valoración de los condicionantes físicos, pero tampoco debe olvidarse la actuación humana como motor de cambios profundos en el paisaje.

En este sentido la distribución espacial demuestra cómo las áreas más accesibles son las que han sufrido más intensamente la acción antrópica, y son las que están colonizadas por las comunidades más degradadas. El factor antropogénico está también en la base de la difusión, aparición o eliminación de muchas especies: las repoblaciones son uno de estos aspectos.

Una prueba de que no sea en toda la sierra la asociación Chamaeropideto Rhamnetum el estadio final de la vegetación, se encuentra en el Barranc del Juano, al N. del llamado «Calvari», donde con una orientación hacia el NE., hay un tupido rodal de pinos carrascos, en cuyo seno, se ha comprobado la existencia de numerosísimos brotes jóvenes en una regeneración de Querceto lentiscetum al amparo del ambiente del pequeño bosquete. Es una señal clara de las posibilidades evolutivas de una sucesión progresiva.

De ello se desprende que aprovechando la fácil evolución de los pinares hasta en condiciones difíciles, se puede dar un primer paso hacia la regeneración de las comunidades clímax.

La inexistencia de referencias más abundantes y en el estado actual de la investigación, se plantea una cuestión que precisa de argumentos más sólidos que la simple especulación: ¿la encina que se recuerda era el último resto de una vegetación que en tiempos bastante lejanos cubría gran parte del territorio? 